\title{
Impacto de un terremoto sobre los animales de compañía en tres distritos de la provincia de Chincha, Perú.
}

The impact of an earthquake on tenure and welfare of animals in Perú.

Silvia Panta ${ }^{1}$, Néstor Falcón ${ }^{1}$, Carlos Caballero² ${ }^{2}$ Miguel Torres ${ }^{3}$

\section{RESUMEN}

Objetivo: Evaluar el impacto del terremoto ocurrido el año 2007 sobre la tenencia y bienestar de los animales de compañía y de crianza de traspatio en tres distritos de la Provincia de Chincha, departamento de Ica-Perú. Metodología: Se desarrolló una encuesta que contenía datos demográficos y de tenencia de animales en el periodo pre y post desastre (2007 y 2012). Además, información de muertes y desapariciones post desastre. Resultados y Conclusiones: Se recolectaron 300 encuestas de las cuales $49 \%$ correspondió a Chincha Alta, $40 \%$ a Pueblo Nuevo y $11 \%$ a Tambo de Mora. El $49 \%$ de encuestados fueron varones y $51 \%$ mujeres. Según las encuestas, antes del terremoto existían 262 perros. De ellos, 27.9\% (73) murieron en el desastre, 26\% (68) desaparecieron y 46.1\% (121) se encontraron vivos. Al momento de la encuesta, se reportó 150 animales, lo que indica una disminución de la población de perros del $42.7 \%$. Respecto a los gatos, ante del terremoto existía 153 animales. De ellos, 24.2\% (37) murieron en el desastre, $23.5 \%$ (36) desaparecieron y $52.3 \%$ (80) se encontraron vivos. Al momento de la encuesta, se reportó 95 animales, representando una disminución de la población del 37.9\%. En ambos casos, la composición etárea varío significativamente $(\mathrm{p}<0.05)$ entre lo encontrado antes del terremoto y la actualidad. En el caso de animales de traspatio, antes del terremoto se registró 777 animales, entre aves, porcinos, bovinos, cuyes, entre otros. De ellos 9.9\% (77) murieron en el desastre, $5 \%$ (39) desaparecieron y $85.1 \%$ (661) se encontraron vivos. Se concluye que el terremoto del 2007 produjo un impacto negativo sobre la población de animales de compañía en la zona, afectando su número y composición etárea, situación que no se observó en los animales de traspatio.

Palabras Claves: terremoto, perros, gatos, Ica, bienestar, crianza de traspatio.

\begin{abstract}
Aim: To evaluate the impact of 2007 earthquake on tenure and welfare of pets and backyard breeding in three districts in the province of Chincha, Ica, Perú. Methods: We developed a survey containing demographic data, tenure of different types of animals during a pre-disaster period (2007) and now (2012), besides information on deaths and disappearances post disaster. Results and Conclusions: 300 surveys were collected, $49 \%$ corresponded to Chincha Alta, $40 \%$ to Pueblo Nuevo and $11 \%$ to Tambo de Mora. $49 \%$ respondents were male and $51 \%$ female. According to surveys, before the earthquake there were 262 dogs. $27.9 \%$ (73) died in the disaster, $26 \%$ (68) disappeared and $46.1 \%$ (121) were found alive. At the time of the survey, 150 animals were reported, which means dog population was decrease by $42.7 \%$. Regarding cats, before the earthquake were 153 animals. $24.2 \%$ (37) died in the disaster, $23.5 \%$ (36) disappeared and 52.3\% (80) were found alive. At the time of the survey, 95 animals were
\end{abstract}

\footnotetext{
${ }^{1}$ Facultad de Medicina Veterinaria y Zootecnia - Universidad Peruana Cayetano Heredia.

${ }^{2}$ Facultad de Medicina Veterinaria y Zootecnia - Universidad Nacional San Luis Gonzaga de Ica.

${ }^{3}$ Facultad de Medicina Veterinaria de la Universidad Agraria de La Habana Fructuoso Rodríguez Pérez.
} 
reported, representing a decrease by $37.9 \%$ in population. Both cases, age composition varied significantly $(\mathrm{p}<$ 0.05 ) between the findings before the earthquake and now. For backyard animals 777 animals were recorded before the earthquake, including birds, pigs, cattle, guinea pigs, among others. 9.9\% (77) of them died in the disaster, 5\% (39) disappeared and $85.1 \%$ (661) were found alive. In conclusion, the earthquake in 2007 had a negative impact on pet population in the area, affecting the number and age composition, which is not observed in backyard animals.

Key Words: Earthquake, dogs, cats, Ica, welfare, backyard breeding.

\section{INTRODUCCIÓN}

Los desastres en el campo de la veterinaria afectan la interacción entre el animal y el hombre en forma directo por medio de la alteración del ecosistema (Ortega et al., 2005), afectando la economía de los productores pecuarios y la salud de la población animal (Henao et al., 2006). El reconocimiento previo de esos efectos puede facilitar un mejor uso de los recursos de salud de la comunidad y fundamentalmente, las comunidades pueden evitar estos riesgos o básicamente prepararse para enfrentarlos (Bello et al., 2004).

Un grupo de animales, muy ligado a las personas, que se ven afectados en casos de desastres son los animales de compañía. Estos son criados y reproducidos con la finalidad de vivir con las personas, con fines educativos, sociales o lúdicos, sin ninguna actividad lucrativa, por lo que la relación con las personas en ocasiones puede ser extremadamente fuerte (Correa et al, 2011).

Esta relación puede llegando a afectar regulaciones de salud pública si es que estas no están consideradas en los planes de rescate. Una muestra de ello se observó en Indiana - USA (1997), en donde la negativa a evacuar la zona afectada por una inundación fue la tenencia de animales de compañía (Sebastián et al., 2001). En contraste, en la Florida - USA, ante la presencia del huracán Floyd, el cuidado de los animales se incluía dentro de las funciones de los equipos de rescate, con la misma importancia que se le daba a la preservación de la seguridad de la familia y la protección del personal mientras trabajaba, aunque no definían claramente de quien era la función (French et al., 2002). En estos equipos participan Médicos Veterinario que utilizan animales adiestrados (perros $\mathrm{u}$ otros), organizar la atención veterinaria y otras medidas de emergencia a los animales afectados (Chávez et al., 1999).

El último terremoto que se produjo en el Perú ocurrió el 15 de agosto de 2007 a las 18.40.57 UTC (18:40:57 hora local), siendo el departamento de Ica, ciudad ubicada al sur de Lima ciudad capital, el epicentro del mismo ( $\left.13^{\circ} 353^{\prime} \mathrm{S} 76^{\circ} 509^{\prime} \mathrm{O}\right)$. La magnitud del evento telúrico fue de 7.0ML (Richter), $7.9 \mathrm{Mw}$ (magnitud momento). El impacto sobre las personas ha sido medido mas no en el caso de los animales de compañía. La necesidad de determinar el mismo permitirá identificar problemas que se pueden encontrar en estas poblaciones en futuras situaciones de desastre y para el cual se debería de estar preparado. Por ello, el objetivo del estudio fue evaluar el impacto del terremoto ocurrido el año 2007 sobre los animales de compañía (expresados en muertos, desaparecidos y sobrevivientes) en tres distritos de la Provincia de Chincha, departamento de Ica-Perú.

\section{MATERIALES Y MÉTODOS}

El estudio se realizó en la ciudad de Chincha ubicada a 200 kilómetros al sur de Lima, sobre los 94 $\mathrm{msnm}$. Esta ciudad tiene una superficie de $238.34 \mathrm{~km}^{2}$ y una proyección de población de 60000 habitantes. La provincia está compuesta de 11 distritos. El estudio considero trabajar en tres distritos: Chincha Alta, Pueblo Nuevo y Tambo de Mora. La elaboración de las encuestas, bases de datos y análisis estadístico se realizó en la Facultad de Veterinaria y Zootecnia de la Universidad Peruana Cayetano Heredia (FAVEZ$\mathrm{UPCH})$.

La población objetivo fueron las familias de los tres distritos seleccionados para el estudio, que hayan habitado la zona durante el terremoto del año 2007. El tamaño de muestra fue definido utilizando la fórmula de comprobación de una proporción para poblaciones desconocidas. Las restricciones fueron las siguientes: proporción referencia de $75 \%$ correspondiente a la proporción de familias que vieron afectadas (según publicaciones periodísticas), nivel de confianza del 95\% y error máximo admisible del 5\%. El tamaño de muestras calculado fue de 288 encuestas.

Para la recolección de información se elaboró un instrumento tipo encuesta el mismo que contenía preguntas abiertas y cerradas (encuesta semiestructurada). Las informaciones recolectadas 
Cuadro 1. Características generales de las personas encuestadas tras el terremoto del 2007, en tres distritos de la provincia de Chincha-Ica, Perú. Encuesta 2012

\begin{tabular}{llll}
\hline \multirow{2}{*}{ Variable } & Estrato & \multicolumn{2}{c}{ Encuestado } \\
\cline { 2 - 4 } Zona & Chincha Alta & Numero & Porcentaje \\
\hline Geográfica & Pueblo Nuevo & 147 & 49 \\
& Tambo de Mora & 120 & 40 \\
Sexo & Masculino & 33 & 11 \\
& Femenino & 147 & 49 \\
Grado & Sin educación escolar & 153 & 51 \\
De & Educación primaria & 3 & 1 \\
Instrucción & Educación secundaria & 28 & 9.3 \\
& Educación superior técnica & 102 & 34 \\
Actividad & Educación superior universitaria & 123 & 41 \\
Laboral & Dependiente & 44 & 14.7 \\
& Independiente & 102 & 34 \\
\hline
\end{tabular}

Cuadro 2. Consecuencias inmediatas del terremoto del 2007 sobre la poblaciones de canes domésticos en tres distritos de la provincia de Chincha- Ica, Perú. Encuesta 2012

\begin{tabular}{|c|c|c|c|c|c|c|c|c|c|}
\hline \multirow{3}{*}{ Especies } & \multirow{3}{*}{$\begin{array}{l}\text { Antes } \\
\mathrm{N}^{\circ}\end{array}$} & \multicolumn{6}{|c|}{ Inmediatamente post terremoto } & \multirow{3}{*}{$\begin{array}{l}\text { Ahora } \\
\mathrm{N}^{\circ}\end{array}$} & \multirow{3}{*}{$\begin{array}{l}\text { Porcentaje de } \\
\text { variación }\end{array}$} \\
\hline & & \multicolumn{2}{|c|}{ Muertos } & \multicolumn{2}{|c|}{ Perdidos } & \multicolumn{2}{|c|}{ Vivos } & & \\
\hline & & $\mathrm{N}^{\circ}$ & $\%$ & $\mathrm{~N}^{\circ}$ & $\%$ & $\mathrm{~N}^{\circ}$ & $\%$ & & \\
\hline Cachorro & 58 & 18 & 24.7 & 13 & 19.1 & 27 & 22.3 & 18 & -69 \\
\hline Joven & 145 & 33 & 45.2 & 35 & 51.5 & 77 & 63.6 & 10 & -93.1 \\
\hline Adulto & 52 & 19 & 26 & 19 & 27.9 & 14 & 15 & 118 & +126.9 \\
\hline Geronte & 7 & 3 & 4.1 & 1 & 1.5 & 3 & 2.5 & 4 & -42.9 \\
\hline TOTAL & 262 & 73 & 27.9 & 68 & 26 & 121 & 46.2 & 150 & -42.7 \\
\hline
\end{tabular}

Cuadro 3. Consecuencias inmediatas del terremoto del 2007 sobre la poblaciones de felinos domésticos en tres distritos de la provincia de Chincha-Ica, Perú. Encuesta 2012

\begin{tabular}{|c|c|c|c|c|c|c|c|c|c|}
\hline \multirow{3}{*}{ Especies } & \multirow{3}{*}{$\begin{array}{l}\text { Antes } \\
\mathrm{N}^{\circ}\end{array}$} & \multicolumn{6}{|c|}{ Inmediatamente post terremoto } & \multirow{3}{*}{$\begin{array}{l}\text { Ahora } \\
\mathrm{N}^{\circ}\end{array}$} & \multirow{3}{*}{$\begin{array}{l}\text { Porcentaje de } \\
\text { variación }\end{array}$} \\
\hline & & \multicolumn{2}{|c|}{ MUERTOS } & \multicolumn{2}{|c|}{ PERDIDOS } & \multicolumn{2}{|c|}{ VIVOS } & & \\
\hline & & $\mathrm{N}^{\circ}$ & $\%$ & $\mathrm{~N}^{\circ}$ & $\%$ & $\mathrm{~N}^{\circ}$ & $\%$ & & \\
\hline Cachorro & 47 & 15 & 40.5 & 6 & 16.7 & 26 & 32.5 & 14 & -70.2 \\
\hline Joven & 75 & 15 & 40.5 & 19 & 52.8 & 41 & 51.3 & 10 & -86.7 \\
\hline Adulto & 28 & 6 & 16.2 & 10 & 27.8 & 12 & 15.0 & 68 & +142.9 \\
\hline Geronte & 3 & 1 & 2.7 & 1 & 2.8 & 1 & 1.3 & 3 & 0 \\
\hline TOTAL & 153 & 37 & 24.2 & 36 & 23.5 & 80 & 52.3 & 95 & -37.9 \\
\hline
\end{tabular}

fueron: datos generales del jefe de familia (edad, sexo, ocupación, distrito de procedencia, ingreso familiar), información acerca de la tenencia de animales de compañía en el periodo julio-agosto del
2007, información del impacto del terremoto del 2007 expresado en animales muertos, desaparecidos y sobreviviente, y la tenencia actual. 
Cuadro 4. Asistencia sanitaria y tipo de alimentación en animales de compañía antes y después del terremoto del 2007 en tres distritos de la provincia de Chincha-Ica, Perú. Encuesta 2012

\begin{tabular}{|c|c|c|c|c|c|c|}
\hline \multirow{3}{*}{$\begin{array}{l}\text { Variable de } \\
\text { Evaluación }\end{array}$} & \multicolumn{3}{|c|}{ Antes (2007) } & \multicolumn{3}{|c|}{ Después (2012) } \\
\hline & \multirow{2}{*}{$\begin{array}{l}\mathrm{N}^{\circ} \text { de } \\
\text { encuestas }\end{array}$} & \multicolumn{2}{|c|}{$\begin{array}{l}\text { Respuestas } \\
\text { afirmativas }\end{array}$} & \multirow{2}{*}{$\begin{array}{l}\mathrm{N}^{\circ} \text { de } \\
\text { encuestas }\end{array}$} & \multicolumn{2}{|c|}{$\begin{array}{l}\text { Respuestas } \\
\text { afirmativas }\end{array}$} \\
\hline & & $\mathrm{N}^{\circ}$ & $\%$ & & $\mathrm{~N}^{\circ}$ & $\%$ \\
\hline Vacuna contra la rabia & 214 & 133 & 62.0 & 139 & 114 & 82.0 \\
\hline Otras vacunas & 214 & 74 & 34.6 & 138 & 69 & 50.0 \\
\hline Desparasitación & 214 & 93 & 43.5 & 139 & 120 & 86.3 \\
\hline Alimento concentrado & 214 & 28 & 13.1 & 139 & 17 & 12.2 \\
\hline
\end{tabular}

Para realizar la encuesta, se contó con el apoyo de estudiantes de Medicina Veterinaria de la universidad local (San Luis Gonzaga de Ica) los cuales fueron capacitados para esta actividad. Los encuestadores leyeron el instrumento y llenaron el mismo de acuerdo a las respuestas que ofrecía el encuestado.

La información obtenida a partir de la encuesta fue transferida a una base de datos en el programa Microsoft Excel y evaluada a través del programa estadístico SPSS 19.0. Los resultados se resumieron utilizando cuadros de frecuencias y las comparaciones de los grupos etáreos antes del terremoto y al momento de realizar el estudio fueron evaluadas mediante la prueba de Chi Cuadrado.

\section{RESULTADOS}

Se recolectaron 300 encuestas de las cuales el $49 \%$ (147) correspondió a habitantes Chincha Alta, 40.0\% (120) a Pueblo Nuevo y 11\% (33) a Tambo de Mora. La caracterización de las personas encuestadas se presenta en el cuadro 1.

Según los encuestados, ante del terremoto existían 262 perros. De ello, 27.9\% (73) murieron en el desastre, $26.0 \%$ (68) desaparecieron y $46.1 \%$ (121) se encontraron vivos. Al momento de la encuesta, se reportó 150 animales, lo que indica una disminución de la población de perros del $42.7 \%$. La composición etárea varío significativamente $(p<0.05)$ entre lo encontrado antes del terremoto y la actualidad.

En caso de los felinos, según los encuestados, ante del terremoto existía 153 animales. De ello, $24.2 \%$ (37) murieron en el desastre, $23.5 \%$ (36) desaparecieron y $52.3 \%(80)$ se encontraron vivos. Al momento de la encuesta, se reportaron 95 animales, lo que representa una disminución de la población del $37.9 \%$. La composición etárea, antes del terremoto y la actualidad, también varío significativamente $(\mathrm{p}<0.05)$ Las consecuencias inmediatas del terremoto del 2007 sobre las poblaciones de canes y felinos domésticos se presentan en detalle en los cuadros 2 y 3 respectivamente. En el cuadro 4 se resume el tipo de asistencia sanitaria y alimentación que se les brindaros a los animales de compañía, antes y después del terremoto.

\section{DISCUSIÓN}

El terremoto del 15 de agosto de 2007 se produjo a las 18:40 hora local, alcanzó una magnitud 7.0 en la escala de Richter y tuvo una duración aproximada de 175 segundos. Chincha, una provincia del departamento de Ica fue la más afectada, después de Pisco. Tras el desastre el gobierno promulgó siete Decretos de Urgencia, con la finalidad de brindar ayuda económica y social a la población damnificada por el impacto del desastre (Defensa Civil, 2007), en los cuales los animales de compañía no estaban considerados.

La cuantificación del impacto de este fenómeno natural sobre la población de animales de compañía ha tratado de ser cubierta por este estudio y para ello se seleccionaron tres distritos usando como criterio la accesibilidad de la misma. El muestreo utilizado fue de conveniencia debido a que el mismo involucró a los jefes de familia que voluntariamente decidieron participar en el estudio respondiendo las encuestas.

El impacto del terremoto en los perros y gatos se puedo medir a partir de los animales reportados como muertos y desaparecidos. En ambos casos los resultados de la encuesta indican que alrededor del $50 \%$ de la población se vio afectada. En caso de los animales desaparecidos, ellos deberían de incluir aquellos que murieron y no se encontró su cadáver, o los que pasaron a formar parte de la población vagabunda. 
La causa de esta desaparición se puede deber a que se desorientaron o huyeron buscando seguridad y no pudieron regresar a sus hogares posteriormente.

En caso de que los perros desaparecidos se convirtieran en vagabundos, estos representarían un potencial problema de salud pública debido al riesgo de accidentes por mordedura, diseminación de enfermedades zoonóticas, contaminación ambiental con heces, además de los problemas de bienestar animal que sufren debido a la baja calidad de vida que llevan.

Los riesgos de mordedura y la transmisión de enfermedades son particularmente importantes en caso de niños quienes representan una población vulnerable. Las consecuencias de estos accidentes se expresan no solo por la lesión producida, sino también por los efectos colaterales, como el impacto emocional en las víctimas, las secuelas estéticas, la transmisión de enfermedades, las complicaciones secundarias (infecciones bacterianas) y el costo que incurren para la comunidad y el Estado (Glausiuss et al., 2000).

En caso de animales muertos, la disposición de los cadáveres es importante, especialmente si es que estos representaban reservorios de enfermedades infecciosas. Western (2004) menciona que en el manejo de los cuerpos, en caso de humanos, se debe tener cuidado con ciertas enfermedades endémicas que constituyen casos especiales cuando se considera la disposición prioritaria de los cadáveres; sin embargo también menciona que la investigación científica no ha podido vincular la presencia de cadáveres como causa de una epidemia en ninguno de los desastres recientes o en situaciones con gran cantidad de personas fallecidas, y tampoco se cuenta con información de este tipo en caso de animales.

Inmediatamente después de producido el terremoto, la ayuda humanitaria incluyo a los animales de compañía. Alrededor de 500 animales entre canes y gatos de la ciudad de Chincha fueron los beneficiarios de cuatro toneladas de alimento balanceado, medicinas y la atención médica veterinaria gratuita brindada por miembros del Colegio Médico Veterinario Departamental de Lima y profesionales voluntarios. La sede del Colegio Médico Veterinario Departamental de Ica se convirtió en el centro de acopio de los donativos que los brigadistas voluntarios de Lima trajeron para ayudar tanto a los Médicos Veterinarios damnificados de Chincha como para los animales.
El Ministerio de salud, además de otros organismos no gubernamentales (ONGs) y miembros de la sociedad civil, brindaron apoyo con diversas campañas como: esterilización, vacunación (especialmente contra la rabia), desparasitación, refugio, primeros auxilios a los animales heridos y alimento a los animales que se encontraban desamparados.

Esto puede explicar la mejora de la asistencia sanitaria post terremoto la misma que se habría interiorizado en los propietarios y que se refleja en una mayor preocupación por vacunar y desparasitar a los perros y gatos en la actualidad. La mejora de la alimentación no ha variado probablemente porque esta representa un gasto continuo para sus propietarios, lo que no estarían dispuestos a asumir, a comparación de los gastos eventuales que representa la vacunación y desparasitación que en muchos casos puede resultar gratuita debido a las campañas que realiza el Ministerio de Salud o las municipalidades (campañas de desparasitación gratuitas).

Estas actividades tienen la ventaja de ayudar a la prevención de enfermedades zoonóticas que pueden transmitir los animales a las personas, pero se hace necesario también sensibilizar a los propietarios para que en el futuro ellos asuman los gastos que corresponden al control sanitario de sus animales cuando las autoridades correspondientes suspendan este tipo de ayuda social.

En cuanto al impacto de las esterilizaciones, estas podrían ser la explicación de que la composición etárea de la población canina y felina haya variado, haciendo que en los dos casos se envejeciera. Debemos tener presente que la etapa adulta de un animal es la más larga y este también sería un factor lo cual en el momento de la toma de encuesta se encontrara una mayor población adulta.

Las consecuencias del terremoto de Ica en el año 2007 es una muestra de la necesidad de formar un organismo multidisciplinario, entre los que deben de considerar a los médicos veterinarios, unidos con Defensa Civil. Todos los países, especialmente los que están en alto riesgo, deben hacer el mejor esfuerzo por fortalecer sus grupos de emergencias. La implementación de planes de contingencia deben ser planificados con tiempo suficiente, estimulando ejercicios de simulacro y entrenamiento. Un desastre requiere de una respuesta inmediata, intensiva y especializada que se efectúa en un breve y limitado período de tiempo. A veces es difícil si no se dispone 
anticipadamente de recursos humanos debidamente capacitados.

\section{CONCLUSIONES}

- El impacto del terremoto se expresó en pérdida de animales de compañía producto de muertes o desapariciones de los mismos.

- El número de animales de compañía ha disminuido y la composición etárea de canes y perros varió (se envejeció) comparando la población reportada antes del terremoto y la actual (2012).

- Producto del apoyo recibido post terremoto se observa una mejora en las condiciones sanitarias de los animales de compañía.

\section{Correspondencia}

Silvia Panta

silvia.panta@upch.pe

\section{REFERENCIAS BIBLIOGRÁFICAS}

1. Bello B, Cruz N, Álvarez M, Chao F, García V, et al. 2004. Medicina de Desastres. La Habana, Cuba. Ed. Ciencias Médicas. 206 p.

2. Chávez P, Fernández A, Álvarez J. 1999. Manual de procedimientos veterinarios para situaciones de desastres naturales hidro-meteorológicos. La Habana, Cuba. Ed. Mar y Pesca 127 p.

3. Correa J, Davis M, Ruffin W, Ebert R, Floyd J. 2011. La compañía del perro y sus beneficios para el ser humano. Alabama Cooperative Extension System. UNP-0058.
4. Defensa Civil. 2007. Características del ámbito nacional afectado. Impacto Socio Económico y Ambiental del Sismo del 15 de Agosto del 2007. Capitulo I. 19 p.

5. French E, Sole M, Byers J. 2002. A comparison of nurses' needs/concerns and hospital disaster plans following Florida's Hurricane Floyd. 28(2):111-7.

6. Glausiuss G, Ascione I, Sehabiague G. 2000. Mordeduras por animales en la edad pediátrica. Arch. Pediatr. Urug. 71: 24-30.

7. Henao S, Urcelay S, Pinto J. 2006. Impacto de las inundaciones sobre las explotaciones ganaderas de la provincia de Valdivia (Chile). Revista CES Medicina Veterinaria y Zootecnia Volumen 1 No. 1. $32 \mathrm{p}$.

8. Instituto Geofísico del Perú. Centro Nacional de Datos Geofísicos. 2002. Geodinámica, Sismicidad y Energía sísmica en Perú. Lima, Perú. Capítulo I 3 p.

9. Ortega C, Villamil L, Cediel N, Rosenfeld C, de Meneghi D, de Rosa M, et al. 2005. Las redes SAPUVET y SPVet: un modelo de integración en materia de salud pública veterinaria entre Europa y América Latina. Revista Panamericana de Salud Pública. 17(1): 60-65.

10. Sebastian E, Philip H, Beck A, Glickman L. Human and Pet-related Risk Factors for Household Evacuation Failure During a Natural Disaster American Journal of Epidemiology 153(7): 659-665.

11. Western K. 2004. Manejo de cadáveres en situaciones de desastre. Consideraciones sanitarias en casos de muertes masivas. Capítulo 3.79 p.

Recibido: 05/06/2014

Aceptado: 18/12/2014 